\section{O consumo alimentar de gestantes adolescentes no Município do Rio de Janeiro}

\author{
Food consumption by pregnant adolescents \\ in Rio de Janeiro, Brazil
}

\author{
1 Centro de Saúde Escola \\ Germano Sinval Faria, Escola \\ Nacional de Saúde Pública, \\ Fundação Oswaldo Cruz, \\ Rio de Janeiro, Brasil. \\ 2 Instituto de Nutrição, \\ Universidade Federal \\ do Rio de Janeiro, \\ Rio de Janeiro, Brasil. \\ 3 Departamento de \\ Epidemiologia e Métodos \\ Quantitativos em Saúde, \\ Escola Nacional de Saúde \\ Pública, Fundação Oswaldo \\ Cruz, Rio de Janeiro, Brasil. \\ Correspondência \\ Denise Cavalcante de Barros \\ Rua Leopoldo Bulhões 1480, \\ Rio de Janeiro, $R J$ \\ 21041-210, Brasil. \\ barrosdc@ensp.fiocruz.br
}

\section{Abstract}

The increase in teenage pregnancy has been viewed with concern by public health experts. Food consumption to help maintain high nutrient demands has been identified as one of the most relevant components. This study aims to present the habitual consumption of food and energy-specific nutrients by pregnant adolescents. A total of 1,180 adolescent mothers were interviewed in maternity hospitals in the City of Rio de Janeiro, and a simplified questionnaire on semi-quantitative frequency of food consumption was applied. Lower consumption of fruit juice, vegetables, and fruits was observed among adolescent mothers over 15. Adolescent mothers classified in the lowest quartile of consumption lack the minimum recommended consumption of energy and nutrients. An inverse association was found between the number of household members and energy and nutrient consumption. Adolescent mothers who received dietary information and changed their eating habits during pregnancy showed better results concerning the consumption of energy and nutrients. Prenatal care was a key factor for improving the results of pregnancy, emphasizing the importance of providing dietary information.

Pregnancy in Adolescence; Prenatal Nutrition; Questionnaires
Denise Cavalcante de Barros 1

Rosângela Alves Pereira 2

Silvana Granado Nogueira da Gama 3

Maria do Carmo Leal 3

\section{Introdução}

A gravidez na adolescência tem sido associada à elevação do risco de baixo peso ao nascer, parto pré-termo e mortalidade infantil 1 . Durante a gestação há um incremento das necessidades nutricionais, e na gestante adolescente este aumento é ainda maior por que se superpõem as demandas referentes ao crescimento da mãe e aquelas relacionadas com o desenvolvimento do feto ${ }^{2}$. Apesar de controvérsias, alguns autores referem uma possível competição de nutrientes entre a mãe adolescente e o feto, podendo levar ao nascimento de crianças com baixo peso, principalmente entre as mais jovens que ainda estão em fase de crescimento acelerado 3 .

Rees et al. 4 assinalam que os fatores sócioeconômicos têm também contribuição relevante na determinação desse risco, independente do efeito da idade materna. Gama et al. 1 também destacam que a pobreza, a má alimentação, a falta de instrução, a marginalização social e hábitos de vida pouco saudáveis, e a ausência de assistência médica durante o pré-natal têm tido um importante papel nesse processo.

A falta de conhecimento sobre alimentação saudável pelas jovens grávidas reflete-se nas suas escolhas alimentares, que são influenciadas por fatores como o apetite aumentado, o "desejo", o paladar acentuado, a conveniência 
e a disponibilidade do alimento e as influências culturais e familiares 5 .

Os distúrbios nutricionais identificados como expressivos na adolescência e na gestação referem-se sobretudo ao consumo de energia, proteína, ferro, folato, cálcio e vitamina A 5,6,7.

A assistência à gestante constitui-se numa excelente oportunidade para a orientação para a saúde em geral e dietética em particular. Gama et al. 8 estudaram uma amostra de gestantes adolescentes no Município do Rio de Janeiro e identificaram a assistência pré-natal como um fator de proteção, minimizador do efeito das desigualdades sócio-econômicas sobre os resultados adversos da gravidez na adolescência.

O presente estudo investigou o consumo alimentar de gestantes adolescentes no Município do Rio de Janeiro, descrevendo os alimentos mais consumidos, a ingestão diária de energia, proteína, ferro, vitamina $\mathrm{C}$, folato e cálcio, e a sua associação com variáveis selecionadas.

\section{Metodologia}

\section{Desenho e população do estudo}

Trata-se de um estudo descritivo do tipo transversal, desenvolvido dentro da investigação $A$ Gravidez na Adolescência como Variável Explicativa do Baixo Peso ao Nascer e Outros Efeitos Adversos no Recém Nascido em Maternidades do Município do Rio de Janeiro, que é um subprojeto do "Estudo da Morbi-mortalidade e da Atenção Peri e Neonatal no Município do Rio de Janeiro", pela Escola Nacional de Saúde Pública (ENSP) da Fundação Oswaldo Cruz (FIOCRUZ), em convênio com a Secretaria Municipal de Saúde do Rio de Janeiro (SMS-RJ). O estudo principal envolveu uma amostra de $10.072 \mathrm{pu}$ érperas que se hospitalizaram em maternidades localizadas no município por ocasião do parto. As maternidades foram agrupadas em três estratos de tamanhos semelhantes. O primeiro estrato continha estabelecimentos municipais e federais, o segundo, estabelecimentos militares, estaduais, filantrópicos e privados conveniados com o SUS e o terceiro, estabelecimentos privados. Maiores detalhes da amostra estão em Leal et al. 9 .

O projeto foi aprovado pela Comissão de Ética para Pesquisa da FIOCRUZ e um Termo de Consentimento Livre e Esclarecido foi assinado pelas mães ou responsáveis pelas adolescentes, após a concordância em participar da pesquisa.

A população a que se refere este artigo foi constituída por 1.228 puérperas adolescentes com idade entre 12 e 19 anos, no período compreendido entre julho de 1999 a março de 2001. Destas, 1.180, 96,0\% da amostra original, foram incluídas na análise. Os motivos das perdas foram: não resposta à parte referente à alimentação $(2,0 \%)$, gestação gemelar $(0,8 \%)$ e consumo calórico estimado considerado improvável, inferior a 600 ou superior a $6.000 \mathrm{kcal}(1,2 \%) 10$.

\section{Coleta de dados e instrumento}

As informações aqui analisadas foram obtidas em dois questionários aplicados no pós-parto imediato; o primeiro, aplicado a todas as puérperas, constava de perguntas sobre as condições socioeconômicas, história reprodutiva e utilização de serviços de saúde; o segundo, específico para as adolescentes, abordava, entre outros aspectos, as relações familiares, estilo de vida e a alimentação durante a gravidez. A coleta de dados foi realizada por profissionais de saúde devidamente treinados e reciclados periodicamente para esta finalidade.

Para avaliar o consumo alimentar, foi utilizado um Questionário de Freqüência de Consumo Alimentar (QFCA) composto por uma lista de 23 alimentos, semiquantitativo, no qual constavam porções padronizadas para cada item, como por exemplo: para arroz a porção padrão era "uma colher de sopa cheia"; para macarrão, "um pegador"; para farinha de mandioca, "uma colher de sopa”; para pão, "um pão francês" ou "uma fatia de pão de forma"; para feijão, "uma concha” 11. Desse questionário constavam as seguintes opções de freqüência de consumo: mais de 3 vezes por dia, de 2 a 3 vezes por dia, duas vez por dia, de 5 a 6 vezes por semana, de 2 a 4 vezes por semana, uma vez por semana, 1 a 3 vezes por mês e nunca ou quase nunca.

A lista de alimentos do QFCA foi obtida com base na redução de um questionário que foi utilizado na investigação do consumo de alimentos da população do Rio de Janeiro, desenvolvida na pesquisa de base populacional "Nutrição e Saúde no Rio de Janeiro”, realizada em 1995-1996 12, que investigou 1.668 domicílios, totalizando cerca de 4.500 indivíduos com mais de 12 anos de idade. Nesse estudo, aplicou-se um QFCA validado, que continha uma lista de oitenta itens, a qual foi elaborada baseando-se em dados do ENDEF 13, tendo sido adicionados itens que teriam sido introduzidos na alimentação habitual da população urbana brasileira após a realização do ENDEF. Sichieri et al. (1998 - dados não publicados) utilizando o procedimento stepwise para regressão linear, desenvolveram uma lista reduzida desse QFCA 
que contemplava os 21 alimentos mais consumidos na dieta habitual da população de adultos de 20 a 60 anos do Município do Rio de Janeiro e que proporcionavam $70 \%$ do consumo alimentar em geral, $74 \%$ do consumo de energia, $82 \%$ de proteína, $83 \%$ de cálcio, $71 \%$ de vitamina C. À esta lista foram adicionados os legumes e macarrão cujo consumo é considerado freqüente entre adolescentes no Município do Rio de Janeiro.

\section{Análise dos dados}

Para estimar o consumo diário de energia, proteína, ferro, vitamina $\mathrm{C}$, folato e cálcio elaborou-se um programa específico que previu, inicialmente, a transformação de cada freqüência relatada em freqüência diária (considerandose o valor 1 quando o alimento era consumido uma vez ao dia, as demais opções de freqüência foram proporcionalmente transformadas em freqüências diárias). Em seguida, o número de porções médias usualmente consumidas foi multiplicado pela freqüência diária, obtendose dessa forma o consumo diário de cada item incluído no QFCA.

O conteúdo de energia e nutrientes de cada alimento foi estimado utilizando-se os dados da Tabela de Composição de Alimentos do Programa de Apoio à Nutrição 14, com exceção do folato que usou os dados publicados por Mahan \& Escott-Stump 15. Para o item legumes, utilizouse a média de energia e nutrientes das hortaliças mais consumidas diariamente (tomate, cenoura, alface, pepino, beterraba) pelos adolescentes investigados na Pesquisa de Nutrição e Saúde no Município do Rio de Janeiro 10.

Estimaram-se as prevalências (e respectivos erros-padrão) de consumo alimentar diário e semanal dos itens incluídos no QFCA. A freqüência de consumo diário de cada alimento foi computada considerando as freqüências: mais de 3 vezes/dia; 2 a 3 vezes/dia; 1 vez/dia e 5 a 6 vezes/semana. Para cálculo do consumo semanal, utilizaram-se as mesmas opções de freqüências computadas para consumo diário, além de 2 a 4 vezes e uma vez por semana.

Foram estimadas as recomendações dietéticas de energia e nutrientes para as gestantes estudadas. Para energia e proteínas adotaramse as recomendações do National Research Council 16. Para os micronutrientes adotou-se a Ingestão Dietética de Referência (IDR), segundo o Institute of Medicine 17,18,19,20.

As co-variáveis incluídas no estudo foram:

- Grupo etário: as mães adolescentes foram categorizadas em dois grupos: de 12-14 anos e de 15 a 19 anos.
- Número de pessoas no domicílio: criaramse dois estratos, até três e quatro ou mais pessoas residentes no domicílio da entrevistada.

- Informações sobre a alimentação em consultas de assistência pré-natal: distribuindo-se as mães em dois grupos, as que receberam informações e as que não receberam informações (inclusive as que não fizeram pré-natal).

- Relato de mudança da alimentação durante a gestação: consideraram-se duas categorias, não relatou e relatou mudança.

\section{Tratamento estatístico}

Estabeleceram-se os quartis das distribuições do consumo de energia e nutrientes durante a gravidez das puérperas adolescentes investigadas, e estimaram-se os valores médios dos quartis para o grupo como um todo e segundo as categorias das variáveis independentes e dependentes estudadas. Utilizou-se o teste do Quiquadrado para testar a dependência entre o consumo de energia e nutrientes e as variáveis consideradas, com nível de significância $<0,05$. O teste não paramétrico de Mann-Whitney foi utilizado para avaliar as diferenças entre o consumo de energia e nutrientes segundo os estratos das co-variáveis consideradas, com nível de significância $>0,05$.

Com a regressão logística, testou-se a associação entre o consumo de energia e as co-variáveis que apresentaram dependência estatística, segundo o teste do Qui-quadrado e/ou cujos estratos mostraram-se distintos, de acordo com o teste de Mann-Whitney. O modelo final teve como variável dependente o consumo de energia categorizado em dois estratos: baixo (que incluiu as gestantes classificadas no primeiro quartil da distribuição do consumo de energia) e alto (incluindo as gestantes classificadas no último quartil dessa distribuição). Estimaram-se as odds ratio (OR), ou razão de chances, e os respectivos intervalos de confiança associados às co-variáveis consideradas. Foram consideradas significativas as OR cujos intervalos de confiança não incluíam a unidade.

\section{Resultados}

Na Tabela 1 são apresentados os alimentos de consumo diário referidos pelas puérperas adolescentes durante a gravidez, segundo o grupo etário. Pelo menos $80 \%$ das entrevistadas referiram consumir diariamente os alimentos básicos, tais como arroz, açúcar, manteiga, pão e feijão. Foi também muito freqüente o consumo de leite, refrigerantes, sucos, legumes e laranja, 
Prevalência diária e semanal de consumo de alimentos pelas puérperas adolescentes durante a gravidez, por grupo etário e total. Município do Rio de Janeiro, Brasil, 2001.

\begin{tabular}{|c|c|c|c|c|c|c|c|c|c|c|c|c|}
\hline \multirow[t]{3}{*}{ Alimentos } & \multicolumn{6}{|c|}{ Consumo diário } & \multicolumn{6}{|c|}{ Consumo semanal } \\
\hline & \multicolumn{2}{|c|}{$10-14$ anos } & \multicolumn{2}{|c|}{ 15-19 anos } & \multicolumn{2}{|c|}{ Total } & \multicolumn{2}{|c|}{ 10-14 anos } & \multicolumn{2}{|c|}{ 15-19 anos } & \multicolumn{2}{|c|}{ Total } \\
\hline & $\%$ & Posto & $\%$ & Posto & $\%$ & Posto & $\%$ & Posto & $\%$ & Posto & $\%$ & Posto \\
\hline Arroz & 94,12 & 2 & 93,35 & 1 & 93,38 & 1 & 100,00 & 1 & 99,11 & 1 & 99,15 & 1 \\
\hline Açúcar & 88,00 & 4 & 91,71 & 2 & 91,55 & 2 & 96,00 & 5 & 96,94 & 3 & 96,90 & 3 \\
\hline Manteiga & 96,00 & 1 & 87,36 & 3 & 87,72 & 3 & 98,00 & 4 & 95,55 & 5 & 95,65 & 5 \\
\hline Pão & 90,20 & 3 & 85,63 & 4 & 85,82 & 4 & 100,00 & 1 & 96,98 & 2 & 97,11 & 2 \\
\hline Feijão & 80,39 & 5 & 78,81 & 5 & 78,88 & 5 & 98,04 & 3 & 96,10 & 4 & 96,18 & 4 \\
\hline Leite & 68,00 & 7 & 74,51 & 6 & 74,15 & 6 & 86,00 & 9 & 88,68 & 8 & 88,57 & 8 \\
\hline Refrigerante & 74,51 & 6 & 63,99 & 7 & 64,36 & 7 & 86,27 & 7 & 89,90 & 7 & 89,66 & 7 \\
\hline Suco & 42,86 & 10 & 56,44 & 8 & 55,86 & 8 & 85,71 & 10 & 88,66 & 9 & 88,53 & 9 \\
\hline Legumes & 49,02 & 8 & 54,04 & 9 & 53,83 & 9 & 82,35 & 11 & 88,62 & 10 & 88,35 & 10 \\
\hline Laranja & 45,10 & 9 & 51,02 & 10 & 50,77 & 10 & 78,43 & 12 & 79,64 & 13 & 79,59 & 13 \\
\hline Banana & 35,29 & 14 & 39,40 & 11 & 39,22 & 11 & 64,71 & 17 & 76,49 & 14 & 75,98 & 14 \\
\hline Queijo & 37,25 & 12 & 37,15 & 12 & 37,16 & 12 & 70,59 & 14 & 69,38 & 15 & 69,43 & 15 \\
\hline Biscoito & 26,53 & 15 & 36,57 & 13 & 36,14 & 13 & 48,98 & 22 & 65,86 & 16 & 65,15 & 16 \\
\hline Frango & 37,25 & 12 & 31,65 & 14 & 31,89 & 14 & 86,27 & 7 & 90,96 & 6 & 90,75 & 6 \\
\hline Chocolate & 41,18 & 11 & 25,63 & 15 & 26,30 & 15 & 70,59 & 14 & 60,45 & 18 & 60,89 & 18 \\
\hline Macarrão & 11,76 & 18 & 18,54 & 16 & 18,24 & 16 & 76,47 & 13 & 80,39 & 12 & 80,22 & 12 \\
\hline Carne & 19,61 & 16 & 14,65 & 17 & 14,87 & 17 & 90,20 & 6 & 85,44 & 11 & 85,64 & 11 \\
\hline Batata frita & 13,73 & 17 & 11,12 & 18 & 11,23 & 18 & 70,59 & 16 & 63,70 & 17 & 64,00 & 17 \\
\hline Ovo & 11,76 & 18 & 7,78 & 19 & 7,96 & 19 & 60,78 & 18 & 59,84 & 19 & 59,88 & 19 \\
\hline Salgadinho & 7,84 & 20 & 4,88 & 20 & 5,01 & 20 & 50,98 & 19 & 47,60 & 22 & 47,66 & 22 \\
\hline Pizza & 2,00 & 21 & 2,59 & 21 & 2,56 & 21 & 34,00 & 23 & 34,82 & 23 & 34,79 & 23 \\
\hline Salsicha & 1,96 & 22 & 2,58 & 23 & 2,56 & 22 & 49,02 & 20 & 48,09 & 21 & 48,13 & 21 \\
\hline Peixe & 0,00 & 23 & 2,59 & 22 & 2,47 & 23 & 49,02 & 20 & 52,63 & 20 & 52,47 & 20 \\
\hline
\end{tabular}

correspondendo a mais da metade das entrevistadas. Analisando por grupos etários, observa-se uma discreta variação entre as menores de 15 e as de 15 a 19 anos. Verifica-se entre as mais jovens um consumo mais freqüente de pão, manteiga, refrigerante, chocolate e salgadinho e menor de sucos, legumes, frutas e biscoitos.

Arroz, pão, açúcar, feijão, manteiga, frango, refrigerante, leite, suco, legumes, carne e macarrão são consumidos pelo menos uma vez por semana, por no mínimo $80 \%$ do grupo investigado. Assinale-se que com exceção de salsicha, salgadinho e pizza, todos os demais itens incluídos no QFCA eram consumidos semanalmente por pelo menos $50 \%$ das entrevistadas. Outra observação relevante é a elevada prevalência do consumo semanal de itens altamente concentrados em gorduras e calorias como batata frita, salgadinho e pizza. As diferenças no consumo semanal de alimentos entre os dois grupos etários seguem a mesma tendência descrita para o consumo diário.
As tabelas apresentadas a seguir não foram discriminadas por faixa etária, pois não ocorreram diferenças estatísticas significantes entre os grupos.

A Tabela 2 apresenta as médias e os erros-padrão de consumo de energia e nutrientes dos quartis, bem como a recomendação média estimada para as gestantes deste estudo. A média de consumo de energia do último quartil durante a gravidez foi 2,5 vezes maior que a do primeiro quartil, demonstrando importantes diferenças no consumo de energia. Em relação ao consumo de proteínas, ferro, cálcio, vitamina $\mathrm{C}$ e folato observa-se, também, uma ampla variação entre o consumo médio no primeiro e no último quartis.

A Tabela 3 apresenta as médias dos quartis de consumo diário de energia e nutrientes, segundo as co-variáveis consideradas neste estudo. O pequeno número de pessoas na família correlacionou-se com pior consumo de energia, proteína, ferro e cálcio. O teste de MannWhitney foi discordante com o teste do Quiquadrado somente para o folato. 
Médias (errros-padrão - EP) dos quartis das distribuições de consumo de energia e nutrientes pelas puérperas adolescentes durante a gravidez $(n=1.180)$. Município do Rio de Janeiro, Brasil, 2001.

\begin{tabular}{|c|c|c|c|c|c|c|c|c|c|}
\hline \multirow[t]{2}{*}{ Energia/nutrientes } & \multicolumn{2}{|c|}{ Quartil 1} & \multicolumn{2}{|c|}{ Quartil 2} & \multicolumn{2}{|c|}{ Quartil 3} & \multicolumn{2}{|c|}{ Quartil 4} & \multirow{2}{*}{$\begin{array}{l}\text { Ingestão } \\
\text { recomendada* }\end{array}$} \\
\hline & Média & EP & Média & EP & Média & EP & Média & EP & \\
\hline Energia (kcal) & 1.224 & 12 & 1.727 & 7 & 2.195 & 9 & 3.149 & 37 & 2.462 \\
\hline Proteína (g) & 41 & 0,5 & 59 & 0,5 & 75 & 0,7 & 109 & 1,5 & 56 \\
\hline Ferro (mg) & 6 & 0,1 & 8 & 0,2 & 12 & 0,2 & 19 & 0,3 & 27 \\
\hline Vitamina C (mg) & 38 & 5 & 106 & 7 & 193 & 9 & 441 & 13 & 80 \\
\hline Folato $(\mu \mathrm{g})$ & 213 & 7 & 347 & 10 & 506 & 12 & 879 & 18 & 600 \\
\hline Cálcio (mg) & 462 & 10 & 696 & 12 & 965 & 15 & 1.488 & 26 & 1.300 \\
\hline
\end{tabular}

* Calculada com base na Recommended Dietary Allowance e Dietary Reference Intake, para a puérpera adolescente padrão da população do estudo.

As variáveis "ter recebido informações sobre alimentação no pré-natal" e "relato de mudança da alimentação durante a gravidez", também mostraram associação com o consumo de energia e todos os nutrientes. $\mathrm{O}$ teste de Mann-Whitney foi concordante com o teste do Qui-quadrado.

Na Tabela 4, observa-se que o risco de baixo consumo de energia $(<1.224 \mathrm{kcal})$ apresentou-se elevado quando a gestante residia em domicílios que tinham menos de 3 pessoas $(\mathrm{OR}=$ 1,9; IC95\%: 1,3-2,6), relatou não ter recebido informações sobre alimentação durante o prénatal $(\mathrm{OR}=1,8$; IC95\%: 1,2-2,7) e referiu não ter mudado a alimentação durante a gravidez $(\mathrm{OR}=$ 1,7; IC95\%: 1,2-2,4).

\section{Discussão}

Neste estudo, foi possível identificar os alimentos mais consumidos na dieta das adolescentes durante a gravidez, além da situação de consumo diário de energia, proteínas, ferro, folato, vitamina $\mathrm{C}$ e cálcio em relação às recomendações nutricionais para a população do estudo. Esta abordagem tem sido útil no reconhecimento do padrão alimentar de populações ou grupos específicos, e, também, na avaliação da associação entre consumo de alimentos com doenças ou outros desfechos, subsidiando a assistência nutricional na promoção, prevenção e intervenção sobre a saúde 12 .

Os dados mostram que as mães adolescentes tinham, durante a gravidez, um consumo freqüente de alimentos de origem animal, como o leite e o frango, e também de outros itens como refrigerantes, açúcar, salgadinho e batata frita. O consumo semanal reflete com mais propriedade as variações intra-individuais na dieta. A análise dos itens alimentares referidos como consumidos pelo menos uma vez por semana revelou uma freqüência elevada de consumo semanal de refrigerantes, batata frita, salgadinho e pizza. Observou-se, ainda, que entre as mães com menos de 15 anos de idade a freqüência do consumo de alimentos menos nutritivos foi mais elevada do que entre aquelas com idades acima de 15 anos, podendo estar relacionado a uma menor exposição à assistência pré-natal e orientação nutricional 21. Estes resultados foram coerentes com as mudanças referidas no padrão da alimentação da população brasileira urbana nos últimos anos, destacandose o aumento no consumo de carnes, laticínios, açúcar refinado e refrigerantes e a diminuição de legumes, verduras e frutas na dieta 22 .

As gestantes adolescentes apresentam um comportamento alimentar monótono e desfavorável para a sua saúde, concordando com os resultados já apresentados para a população de adolescentes do Município do Rio de Janeiro ${ }^{10}$. Story \& Moe 23 , estudando gestantes adolescentes americanas encontraram comportamento alimentar semelhante ao de adolescentes não grávidas, como o consumo freqüente de lanches ricos em açúcar e gorduras e preferência na escolha de alimentos prontos para o consumo.

O consumo de energia durante a gestação das mães entrevistadas foi inferior à ingestão recomendada para cerca de metade das adolescentes. Vários fatores podem estar influenciando, como por exemplo, as necessidades nutricionais aumentadas pela gravidez e a persistência das demandas da maturação e crescimento físico ${ }^{3}$. A adoção de estilos de vida e padrões de estética corporal que induzem a uma restrição alimentar, constituem-se num outro fator que compromete o estado nutricional das 
Tabela 3

Média dos quartis das distribuições do consumo de energia e nutrientes das puérperas adolescentes durante a gravidez, segundo número de pessoas no domicílio ( $n=1.179)$, informações recebidas sobre alimentação no pré-natal ( $n=1.178$ ) e relato de mudança de alimentação durante a gestação $(n=1.173)$. Município do Rio de Janeiro, Brasil, 2001.

\begin{tabular}{|c|c|c|c|c|c|c|c|c|c|c|c|c|}
\hline \multirow{2}{*}{$\begin{array}{l}\text { Quartil de } \\
\text { consumo } \\
\text { de energia } \\
\text { e nutrientes }\end{array}$} & \multicolumn{4}{|c|}{ Número de pessoas no domicílio } & \multicolumn{4}{|c|}{$\begin{array}{c}\text { Informações sobre alimentação } \\
\text { no pré-natal }\end{array}$} & \multicolumn{4}{|c|}{ Mudou a alimentação } \\
\hline & até 3 & 4 ou mais & $\chi^{2}(p)$ & $\begin{array}{l}\text { Mann- } \\
\text { Whitney (p) }\end{array}$ & Sim & Não & $\chi^{2}(\mathrm{p})$ & $\begin{array}{l}\text { Mann- } \\
\text { Whitney (p) }\end{array}$ & Sim & Não & $\chi^{2}(p)$ & $\begin{array}{l}\text { Mann- } \\
\text { Whitney (p) }\end{array}$ \\
\hline \multicolumn{13}{|l|}{ Energia (kcal) } \\
\hline $1 \underline{0}$ & 1.245 & 1.188 & 0,000 & 0,002 & 1.232 & 1.207 & 0,016 & 0,001 & 1.252 & 1.201 & 0,001 & 0,000 \\
\hline 2으 & 1.728 & 1.725 & & & 1.729 & 1.721 & & & 1.719 & 1.735 & & \\
\hline 3으 & 2.184 & 2.205 & & & 2.204 & 2.164 & & & 2.198 & 2.190 & & \\
\hline 4으 & 3.126 & 3.171 & & & 3.163 & 3.097 & & & 3.230 & 3.022 & & \\
\hline \multicolumn{13}{|l|}{ Proteína (g) } \\
\hline 1 으 & 42 & 41 & 0,000 & 0,000 & 42 & 40 & 0,0023 & 0,002 & 42 & 41 & 0,000 & 0,000 \\
\hline 2으 & 58 & 59 & & & 59 & 59 & & & 59 & 59 & & \\
\hline 3으 & 75 & 75 & & & 75 & 75 & & & 75 & 75 & & \\
\hline 4으 & 108 & 111 & & & 110 & 106 & & & 111 & 106 & & \\
\hline \multicolumn{13}{|l|}{ Ferro (mg) } \\
\hline 1으 & 6 & 6 & 0,000 & 0,001 & 6 & 6 & 0,024 & 0,005 & 6 & 6 & 0,000 & 0,000 \\
\hline 2으 & 8 & 8 & & & 8 & 9 & & & 8 & 9 & & \\
\hline 3으 & 12 & 12 & & & 12 & 12 & & & 12 & 12 & & \\
\hline 4으 & 18 & 19 & & & 19 & 18 & & & 19 & 18 & & \\
\hline \multicolumn{13}{|c|}{ Vitamina C (mg) } \\
\hline 1 으 & 38 & 38 & 0,082 & 0,631 & 38 & 39 & 0,002 & 0,000 & 40 & 36 & 0,000 & 0,000 \\
\hline 2oㅇ & 106 & 106 & & & 105 & 111 & & & 106 & 106 & & \\
\hline 3으 & 194 & 192 & & & 192 & 196 & & & 196 & 189 & & \\
\hline 4으 & 436 & 446 & & & 441 & 442 & & & 457 & 406 & & \\
\hline \multicolumn{13}{|l|}{ Folato $(\mu \mathrm{g})$} \\
\hline 1으 & 211 & 217 & 0,385 & 0,037 & 215 & 209 & 0,015 & 0,007 & 213 & 214 & 0,000 & 0,000 \\
\hline 20 & 343 & 350 & & & 346 & 350 & & & 345 & 348 & & \\
\hline 3으 & 509 & 503 & & & 509 & 497 & & & 507 & 505 & & \\
\hline 4으 & 908 & 849 & & & 891 & 833 & & & 902 & 828 & & \\
\hline \multicolumn{13}{|l|}{ Cálcio (mg) } \\
\hline 10 & 459 & 465 & 0,025 & 0,005 & 469 & 444 & 0,002 & 0,000 & 477 & 451 & 0,000 & 0,000 \\
\hline 2oㅇ & 694 & 698 & & & 694 & 700 & & & 705 & 686 & & \\
\hline 3으 & 965 & 965 & & & 966 & 965 & & & 967 & 962 & & \\
\hline 4으 & 1.485 & 1.491 & & & 1.489 & 1.486 & & & 1.522 & 1.418 & & \\
\hline
\end{tabular}

adolescentes 24. Paralelo a isto, as condições socioeconômicas precárias e a falta de aceitação ou apoio na gravidez podem agravar o consumo inadequado de alimentos 23 .

Em relação à proteína, o consumo foi adequado, concordando com estudos nacionais, realizados inclusive na cidade do Rio de Janeiro, que mostram o déficit deste nutriente restrito a grupos populacionais que se encontravam em situação de extrema pobreza ou que estavam fazendo uso de dietas restritas, como as vegetarianas 10,25 .

A média de consumo de vitamina $\mathrm{C}$ excedeu a ingestão recomendada nos três últimos quartis, coerente com o consumo de sucos de frutas, laranja e legumes entre os dez primeiros alimentos mais referidos pelas mães adolescentes. Outros estudos realizados em grandes centros urbanos brasileiros encontraram níveis ótimos de ingestão deste nutriente 10,24,26. 
Risco de baixo consumo de energia, de acordo com as co-variáveis incluídas no modelo de regressão logística comparando o 1 o e o 4o quartis do consumo de energia das puérperas adolescentes durante a gravidez ( $n=588$ ). Município do Rio de Janeiro, Brasil, 2001.

\begin{tabular}{|c|c|c|c|}
\hline Variáveis & OR & IC95\% & p valor \\
\hline Número de pessoas no domicílio (categoria de risco: até 3 pessoas) & 1,9 & $1,3-2,6$ & 0,000 \\
\hline Informação de mudança da alimentação durante a gravidez (categoria de risco: não mudou a alimentação) & 1,7 & $1,2-2,4$ & 0,002 \\
\hline Informações sobre alimentação durante o pré-natal (categoria de risco: não recebeu informação) & 1,8 & $1,2-2,7$ & 0,003 \\
\hline
\end{tabular}

OR $=$ odds ratio; IC95\% = intervalo de confiança de $95 \%$.

Os níveis de ferro foram insuficientes para todos os quartis de consumo. Este era um resultado esperado, tendo em vista que a carência deste nutriente é um problema nutricional de grande relevância na população brasileira, em particular nas crianças e gestantes 22 . A carne vermelha, considerada uma boa fonte de ferro, não aparece entre os alimentos mais consumidos diariamente, embora tenha uma participação considerável no consumo semanal das gestantes adolescentes. Estes resultados foram corroborados por outros autores 5,10,27.

O consumo de folato durante a gravidez esteve abaixo da recomendação para a maior parte das adolescentes, porém, se comparado ao ferro, a situação foi melhor.

Neste estudo, ter encontrado um consumo insuficiente de ferro e folato, não permite inferir sobre a real situação de carência destes nutrientes nesta população porque a sua suplementação faz parte das rotinas da assistência pré-natal 28.

Para o cálcio, observa-se que o consumo foi também insuficiente durante a gestação, muito embora o leite e o queijo, importantes fontes de cálcio, apareçam entre os 12 alimentos mais consumidos. Uma possível explicação é a diminuição da quantidade consumida em substituição, na atualidade, por lanches que incluem refrigerantes, sucos, salgadinhos e batata frita, em particular nesse grupo etário 7,29. Outros estudos revelaram que durante a gestação as adolescentes tenderam a aumentar o consumo de leite, mas ainda assim a ingestão de cálcio na dieta permaneceu abaixo dos níveis recomendados 6 .

É importante destacar que apesar dos alimentos básicos, considerados excelentes fontes calóricas, terem sido referidos por grande proporção das entrevistadas, isso não significou o consumo de uma dieta adequada em energia, mesmo quando se considera que o instrumento utilizado estima cerca de $70 \%$ do consumo de alimentos. O mesmo se repete para o con- sumo freqüente de alimentos ricos em ferro e folato, como o feijão e carnes, e em cálcio, como o leite e o queijo. Estes resultados, como os de outros estudos, revelam que estes alimentos apesar de fazerem parte da dieta habitual das adolescentes grávidas, não são consumidos em quantidades suficientes para atender às suas necessidades dietéticas diárias 24,26.

Observou-se que o risco de consumo insuficiente de energia apresentou-se elevado quando a gestante morava em domicílios com até três pessoas, quando não recebeu informações sobre dieta no pré-natal e informou não ter modificado a sua alimentação durante a gestação.

Mais de $80 \%$ das mães que moravam em domicílios com até três pessoas, viviam só com o marido/companheiro e filho, enquanto que entre aquelas que moravam em domicílios com quatro ou mais pessoas, $60 \%$ viviam com o marido/companheiro, no domicílio dos pais. No primeiro grupo, com freqüência, os chefes de família são também adolescentes, constituindo-se em uma parcela com menor qualificação profissional e, portanto, indivíduos envolvidos com atividades que geram menor renda 21 .

A literatura refere que condições sócio-econômicas desfavoráveis produzem piores resultados na saúde da população em geral e quanto maior a renda, maior o poder de compra e acesso a uma alimentação variada 22 .

As gestantes adolescentes que receberam informações sobre alimentação durante o prénatal e as que informaram mudança de alimentação durante a gestação tiveram melhores resultados no consumo de energia e nutrientes. Estes achados encaminham para o reconhecimento do acompanhamento pré-natal como um momento importante de apreensão de informações, que efetivamente podem estar contribuindo para melhores resultados na dieta alimentar e da gestação. Ter comparecido à consulta no pré-natal e não ter recebido informação, ou mesmo, ter recebido e não ter apre- 
endido, pode ser compreendido neste estudo como uma oportunidade perdida.

Concluindo, pode-se dizer que são múltiplas as dimensões que influenciam o comportamento alimentar da adolescente durante a gestação, desde condições sócio-econômicas, biológicas, comportamentais e de assistência à saúde. Destaca-se o papel que cumpre a assistência pré-natal, na aquisição de hábitos ali- mentares adequados à gestação e neste ciclo de vida, que podem ficar incorporados à vida adulta $4,23,27$.

Os resultados apresentados apontam para a necessidade de estudos mais compreensivos, que detalhem com maior precisão formas mais adequadas de repasse de conhecimento pelos profissionais de saúde sobre a alimentação, para as grávidas adolescentes.

\section{Resumo}

O crescimento da gravidez na adolescência é visto com preocupação pela área de saúde pública. O consumo de alimentos que atendam a alta demanda de $\mathrm{nu}$ trientes durante a gestação tem sido identificado como um componente relevante. O objetivo do estudo foi conhecer o consumo habitual dos alimentos e de energia e nutrientes entre gestantes adolescentes. Foram entrevistadas 1.180 mães adolescentes em maternidades do Município de Rio de Janeiro e aplicado um Questionário de Freqüência de Consumo Alimentar semiquantitativo simplificado. Os alimentos referidos por pelo menos $50 \%$ delas foram arroz, açúcar, manteiga, pão, feijão, leite, refrigerante, sucos, legumes e laranja. Observa-se um menor consumo de sucos, legumes e frutas entre as menores de 15 anos. No primeiro quartil de consumo, a ingestão mínima recomendada de energia, ferro, folato, vitamina C e cálcio não foi alcançada. Encontrou-se uma associação inversa entre o número de pessoas no domicílio e o consumo de energia e nutrientes. O relato de informação sobre alimentação no pré-natal e a informação de mudança de alimentação durante a gravidez esteve associado a um melhor consumo de energia e nutrientes. A atenção pré-natal mostrou-se como um cuidado fundamental para melhores resultados na gestação.

Gravidez na Adolescência; Alimentação na Gravidez; Questionários

\section{Colaboradores}

D. C. Barros realizou a revisão bibliográfica sobre o tema, elaborou a metodologia, conduziu a análise e interpretação dos dados, sendo a principal responsável pela redação do artigo. R. A. Pereira participou da construção do banco de dados de consumo alimentar, contribuiu na análise e interpretação dos dados e redação do artigo. S. G. N. Gama colaborou na revisão da literatura, construção do banco de dados epidemiológicos e revisão final do artigo. M. C. Leal participou da elaboração da metodologia, análise, discussão dos resultados e da redação e revisão final do artigo. 


\section{Referências}

1. Gama SGN, Szwarcwald CL, Leal MC. Experiência de gravidez na adolescência, fatores associados e resultados perinatais entre puérperas de baixa renda. Cad Saúde Pública 2002; 18:153-61.

2. Scholl TO, Hediger ML, Schall JI. Maternal growth and fetal growth: pregnancy course and outcome in Camden study. Ann NY Acad Sci 1997; 817:292-301.

3. Tanner JM. Fetus into men: physical growth from conception to maturity. Cambridge: Harvard University Press; 1978.

4. Rees JM. Overview: nutrition for pregnant and childbearing adolescents. Ann N Y Acad Sci 1997; 817:241-5.

5. Gambardella AMD, Frutuoso MFP, Franchi C. Prática alimentar de adolescentes. Revista de $\mathrm{Nu}$ trição 1999; 12:55-63.

6. Gutierrez Y, King JC. Nutrition during teenage pregnancy. Pediatr Ann 1993; 22:99-108.

7. Story M, Moe J. Eating behaviors and nutritional implications. In: Story M, Stang J, editors. Nutrition and the pregnant adolescent: a practical reference guide. Minneapolis: School of Public Health, University of Minnesota; 2000. p. 47-54.

8. Gama SGN, Szwarcwald CL, Leal MC, Theme Filha MM. Gravidez na adolescência como fator de risco para o baixo peso ao nascer no Município do Rio de Janeiro, 1996 a 1998. Rev Saúde Pública 2001; 35:74-80.

9. Leal MC, Gama SGN, Campos MR, Cavalini LT, Garbayo LS, Brasil CLP, et al. Fatores associados à morbi-mortalidade perinatal em uma amostra de maternidades públicas e privadas do Município do Rio de Janeiro, 1999-2001. Cad Saúde Pública 2004; 20 Suppl 1:S20-33.

10. Andrade RG. Consumo alimentar de adolescentes no Município do Rio de Janeiro, 1995-1996 [Dissertação de Mestrado]. Rio de Janeiro: Instituto de Nutrição, Universidade Federal do Rio de Janeiro; 2000.

11. Sichieri R. Consultoria para desenvolvimento de um questionário simplificado de consumo alimentar. Rio de Janeiro: Universidade do Estado do Rio de Janeiro; 1998.

12. Sichieri R. Epidemiologia da obesidade. Rio de Janeiro: Eduerj; 1998.

13. Fundação Instituto Brasileiro de Geografia e Estatística. Consumo alimentar e antropometria. Regiões I e II. Estudo Nacional da Despesa Familiar - ENDEF. Rio de Janeiro: Fundação Instituito Brasileiro de Geografia e Estatística; 1993.

14. Escola Paulista de Medicina. Programa de apoio à decisão em nutrição, versão 2.5. São Paulo: Universidade Federal de São Paulo; 1995.

15. Mahan LK, Escott-Stump S. Apêndice 47. In: Mahan LK, Escott-Stump S, organizadores. Krause: alimentos, nutrição e dietoterapia. 9ạ Ed. São Paulo: Roca; 1998. p. 1110-22.

16. National Research Council. Recommended dietary allowances. 10th Ed. Washington, DC: National Academy Press; 1989.

17. Institute of Medicine. Dietary reference intakes for, calcium, phosphorus, magnesium, vitamin D, and fluoride. Washington, DC: National Academy Press; 1999.
18. Institute of Medicine. Dietary reference intakes for vitamin C, vitamin E, selenium, and carotenoids. Washington, DC: National Academy Press; 2000.

19. Institute of Medicine. Dietary reference intakes for thiamin, riboflavin, niacin, vitamin B6, folate, vitamin B12, pantothenic acid, biotin, and chlorine. Washington, DC: National Academy Press. 2000.

20. Institute of Medicine. Dietary reference intakes for vitamin A, vitamin $\mathrm{K}$, arsenic, boron, chromium, copper, iodine, iron, manganese, molybdenum, nickel, silicon, vanadium, and zinc. Washington, DC: National Academy Press; 2001.

21. Costa COM, Santos CAS, Sobrinho CL, Freitas JO, Ferreira KASL. Indicadores Materno-Infantis na adolescência e juventude: sociodemográfico, prénatal, partos e nascidos-vivos. J Pediatr (Rio J) 2001; 77:235-42.

22. Monteiro CA, Mondini L, Costa RBL. Mudanças na composição e adequação nutricional da dieta familiar nas áreas metropolitanas do Brasil entre 1988-1996. In: Monteiro CA, organizador. Velhos e novos males da saúde no Brasil: a evolução do país e de suas doenças. 2a Ed. São Paulo: Editora Hucitec/Núcleo de Pesquisas Epidemiológicas em Nutrição e Saúde, Universidade de São Paulo; 2000. p. 359-70.

23. Story M, Alton I. Nutritional issues and adolescent pregnancy. Nutr Today 1995; 30:142-51.

24. Eisenstein E, Coelho KSC, Coelho SC, Coelho MASC. Nutrição na adolescência. J Pediatr 2000; 76 Suppl 3:S263-74.

25. Meirelles CM. Avaliação dietética e antropométrica de adolescentes vegetarianas e onívoras [Dissertação de Mestrado]. Rio de Janeiro: Instituto de Nutrição Josué de Castro, Universidade Federal do Rio de Janeiro; 1999.

26. Machado NO. Caracterização nutricional de uma população de mães adolescentes [Dissertação de Mestrado]. São Paulo: Departamento de Nutrição, Faculdade de Saúde Pública, Universidade de São Paulo; 2001.

27. Beard JL. Iron deficiency: assessment during pregnancy and its importance in pregnant adolescents. Am J Clin Nutr 1994; 59 Suppl:502S-10S.

28. United Nations Children's Fund. Preventing iron deficiency in women and children: technical consensus on key issues and resources for programme advocacy, planning and implementation. New York: United Nations Children's Fund/United Nations University/World Health Organization; 1998.

29. Tobelmann RC, Marquart L, Albertson AM. Estimated dietary calcium intake and food sources for adolescent females: 1980 to 1992. J Adolesc Health 1997; 20:20-6.

Recebido em 08/Out/2002

Versão final reapresentada em 25/Jun/2003

Aprovado em 08/Out/2003 